\title{
Social Learning Requires Plasticity Enhanced by Fluoxetine Through Prefrontal Bdnf-TrkB Signaling to Limit Aggression Induced by Post-Weaning Social Isolation
}

\author{
Éva Mikics',4, Ramon Guirado 2,4, Juzoh Umemori², Máté Tóth', László Biró', Christina Miskolczi', \\ Diána Balázsfi' ', Dóra Zelena', Eero Castrén*,2, József Haller ${ }^{1,5}$ and Nina N Karpova ${ }^{2,3,5}$ \\ 'Department of Behavioral Neurobiology, Institute of Experimental Medicine, Budapest, Hungary; ${ }^{2}$ Neuroscience Center, University of Helsinki, \\ Helsinki, Finland; ${ }^{3}$ School of Pharmaceutical Sciences of Ribeirão Preto, University of São Paulo, Avenida do Café sn, Ribeirão Preto, Brazil
}

\begin{abstract}
Escalated or abnormal aggression induced by early adverse experiences is a growing issue of social concern and urges the development of effective treatment strategies. Here we report that synergistic interactions between psychosocial and biological factors specifically ameliorate escalated aggression induced by early adverse experiences. Rats reared in isolation from weaning until early adulthood showed abnormal forms of aggression and social deficits that were temporarily ameliorated by re-socialization, but aggression again escalated in a novel environment. We demonstrate that when re-socialization was combined with the antidepressant fluoxetine, which has been shown to reactivate juvenile-like state of plasticity, escalated aggression was greatly attenuated, while neither treatment alone was effective. Early isolation induced a permanent, re-socialization-resistant reduction in Bdnf expression in the amygdala and the infralimbic cortex. Only the combined treatment of fluoxetine and re-socialization was able to recover Bdnf expression via epigenetic regulation. Moreover, the behavior improvement after the combined treatment was dependent on TrkB activity. Combined treatment specifically strengthened the input from the ventral hippocampus to the mPFC, suggesting that this pathway is an important mediator of the beneficial behavioral effects of the combined psychosocial and pharmacological treatment of abnormal aggression. Our findings suggest that synergy between pharmacological induction of plasticity and psychosocial rehabilitation could enhance the efficacy of therapies for pathological aggression. Neuropsychopharmacology (2018) 43, 235-245; doi:I0.1038/npp.2017.I42; published online 9 August 2017
\end{abstract}

\section{INTRODUCTION}

Violence and crime are strongly associated with early-life adversities and social neglect during sensitive periods of early life. Although receiving little public attention, early social neglect can be even more detrimental to children than sexual or physical abuse (Gilbert et al, 2009). Although aggression-related disorders recurrently associated with early stressors (Haller et al, 2014) do respond to psychotherapy and pharmacotherapy, their efficacy is rather modest, which makes the development of novel treatment strategies imperative (Coccaro et al, 2009; Society, 2013; Woolfenden et al, 2001).

Previously, we established the model post-weaning social isolation to approximate early social neglect (Toth et al, 2008; Toth et al, 2011) and showed that this model can be used to investigate the mechanisms underlying early adversity-

\footnotetext{
*Correspondence: Dr E Castrén, Neuroscience Center, University of Helsinki, PO Box 56, Helsinki 000 I4, Finland, Tel: +358 505207974 , Fax: +358 919157 620, E-mail: eero.castren@helsinki.fi

${ }^{4}$ The first two authors contributed equally to this work.

${ }^{5}$ The last two authors contributed equally to this work.

Received II November 20 16; revised 26 June 20 17; accepted 28 June 2017; accepted article preview online 7 July 2017
}

induced aggression and to explore novel possibilities of intervention (Sandi and Haller, 2015). Rats submitted to this model deliver substantially more biting attacks to opponents than controls do and, in addition, attacks are preferentially aimed at vulnerable body parts of opponents (head, throat, and belly; Toth et al, 2008; Toth et al, 2011). This behavioral profile is consistent with that characterized in recent literature as escalated or abnormal aggression, and is believed to model aggression-related psychopathologies under laboratory conditions (Miczek et al, 2013). Re-socialization, a laboratory model for behavioral therapy (Miczek et al, 2013), fails to correct the post-weaning social isolation-induced escalation of aggression, confirming the assumption that this paradigm induces long-term changes in emotional processing (Pascual et al, 2006; Wright et al, 1991). These findings suggest that the treatment of developmentally induced aggression problems requires novel approaches.

Sensitive periods of postnatal organization of brain connectivity have been most thoroughly studied in the mammalian sensory systems. In the primary visual cortex, monocular deprivation during the juvenile critical period brings about a drastic loss of responsiveness to stimulation through the deprived eye, whereas no effects on ocular dominance are seen when deprivation takes place after the 
closure of the sensitive period (Hubel et al, 1977). Recent findings suggest that juvenile-like sensitive periods can be reactivated in the adult brain by several means (Bavelier et al, 2010; Sale et al, 2009). Specifically, the antidepressant fluoxetine has been shown to reactivate a critical periodlike plasticity in the visual cortex (Maya Vetencourt et al, 2008) and in the fear circuitry (Karpova et al, 2011). Fluoxetine treatment brings about functional changes when it is combined with environmental manipulations, such as monocular deprivation and fear extinction, but not when the drug is given alone.

Here we hypothesized that the inefficacy of re-socialization in post-weaning social isolation-induced aggression might reflect a closure of early sensitivity period for social learning. We have taken advantage of the possibility to reopen sensitivity periods of heightened plasticity by fluoxetine treatment to test whether juvenile-like plasticity might enhance receptiveness to novel social experiences and thereby allow re-socialization to achieve long-lasting restorative effects. We have further hypothesized that epigenetic changes in the expression of key genes may underlie the closure of critical periods. Brain-derived neurotrophic factor Bdnf has a critical role in neuronal plasticity (Karpova et al, 2011; Maya Vetencourt et al, 2008) and neuronal network structural remodeling (Guirado et al, 2014) induced by fluoxetine. To provide more insight into potential mechanisms by which the anti-aggression treatment exerts its favorable effects, we investigated the expression of activity-dependent Bdnf transcripts 1 and 4 in the aggression circuitry (Siegel et al, 2007): the infralimbic (IL) and prelimbic (PrL) areas of the medial prefrontal cortex (mPFC), the medial (MeA) and central (CeA) amygdala, the hypothalamic attack area, and the lateral hypothalamus (LH). The lack of Bdnf variant transcribed from promoter 1 enhances aggression in mice (Maynard et al, 2016), whereas promoter 4-driven Bdnf transcript is critical for plasticity in the prefrontal cortex (Sakata et al, 2009). Furthermore, we studied the network structural remodeling in the main areas of the aggression circuitry, especially focusing on parvalbumin-containing interneurons encased by perineuronal nets (PV-PNN neurons), since previous studies have implicated these neurons as regulators of juvenile-like plasticity (Karpova et al, 2011).

\section{MATERIALS AND METHODS}

\section{Animals}

We studied male Wistar rats (Charles-River) from the breeding facility of the Institute of Experimental Medicine (Budapest, Hungary). Food and water were available ad libitum. Rats were maintained at temperature $22 \pm 1{ }^{\circ} \mathrm{C}$ and relative humidity $60 \pm 10 \%$, in a light cycle of $12: 12 \mathrm{~h}$ (lights off at 0800 hours). After weaning at the age of 21 days, rats were randomly assigned to social or isolation rearing in cages measuring $42 \times 26 \times 19 \mathrm{~cm}$. When socially housed rats reached the weight of $200 \mathrm{~g}$, they were moved to Makrolon cages measuring $60 \times 38 \times 19 \mathrm{~cm}$. The weight of subjects was $400-450 \mathrm{~g}$ at the age of 11 weeks, when behavioral testing started. In resident-intruder tests, we used male intruders coming from the same source and weighing $\approx 300 \mathrm{~g}$. These rats were housed in groups of six but otherwise maintained under similar conditions. Intruders were used twice; their pairing with residents was randomized such that no resident encountered the same intruder twice. All experiments were carried out in accordance with the European Communities Council Directive of November 24, 1986 (86/609/EEC) and reviewed and approved by the Animal Welfare Committee of the Institute of Experimental Medicine.

\section{Experimental Design}

Post-weaning social isolation and first testing for aggressiveness. Upon weaning, pups were either maintained in individual cages for 8 weeks (post-weaning social isolation; $N=80$ ) or in social groups of four males (social rearing, $N=40$ ). To eliminate litter effects, males from all litters were randomly assigned to social or isolation rearing. The members of socially reared groups came from different litters. To check for the behavioral effects of post-weaning social isolation, rats were tested for aggressiveness in the first resident-intruder test (Test1) on the eighth post weaning (eleventh postnatal) week.

Re-socialization and pharmacological treatment. After the first resident-intruder test (Test1), isolation-reared rats were either returned to their individual cages (isolation; $N=40$ ) or were introduced into social groups of four isolation-reared rats (re-socialization; $N=40$ ). Half of both groups, isolated and re-socialized, were treated with fluoxetine, whereas the other half served as controls for pharmacological treatment and received normal drinking water. Fluoxetine (fluoxetine hydrochloride; Sigma-Aldrich, Budapest) was administered in drinking water at the concentration $0.2 \mathrm{mg} / \mathrm{ml}$. On the basis of regularly recorded fluid intakes and body weights, the average fluoxetine intake was $5.9 \pm 0.1 \mathrm{mg} / \mathrm{day} / \mathrm{rat}(\sim 14 \mathrm{mg} / \mathrm{kg})$. Under similar conditions, fluoxetine significantly increased neural plasticity in rats (Maya Vetencourt et al, 2008; Tiraboschi et al, 2013). Fluoxetine was added to drinking water after the 1-h-long observation period, ie, it did not influence behavior right after re-socialization. Together, the following four treatment groups were studied: (1) 'Isolation'; maintained in isolation after the first resident-intruder test (Test1), no fluoxetine treatment; (2) 'Re-socialization'; re-socialized after Test1, no fluoxetine treatment; (3) 'Isolation+fluoxetine'; maintained in isolation after Test1, fluoxetine treatment; (4) 'Re-socialization+fluoxetine'; re-socialized after Test1, fluoxetine treatment. Re-socialization and fluoxetine administration lasted for 3 weeks. The social behavior in newly formed groups (Re-socialization and Re-socialization+ fluoxetine) was monitored throughout.

Socially reared rats were regrouped after Test1 in a way that at least two rats in the newly formed groups were unfamiliar to each other. This procedure mimicked re-socialization of isolation-reared rats. Half of socially reared rats were treated with fluoxetine, which resulted in two groups: (1) 'Regrouping'; regrouped after Test1 and (2) 'Regrouping+fluoxetine'; regrouped and treated with fluoxetine. These groups served as controls for evaluating behavioral and molecular changes observed in rats reared in social isolation. They were killed at the end of the treatment period, in alternation with isolation-reared rats. 


\section{Experimental Procedures}

The resident-intruder test. Rats were transferred into individual cages $(22 \times 38 \times 28 \mathrm{~cm})$ for 3 days prior to testing to allow for the emergence of territorial behavior. The test consisted in placing a smaller intruder into the subject's home cage for $20 \mathrm{~min}$ in the early hours of the dark period under dim red illumination (provided by two $40 \mathrm{~W}$ red bulbs placed on the ceiling of the experimental room). Behavior was video-recorded and later analyzed at low speed, frameby-frame when necessary, by an experimenter blind to treatments. We recorded the number of biting attacks, their intensity, and targets on the body of opponents according to screening protocols published earlier (Toth et al, 2008; Toth et al, 2011; also see Supplementary Information). Attack counts and their intensity were used to characterize the level of aggressiveness, whereas attack targeting toward vulnerable parts was used to differentiate normal (speciesspecific) from abnormal attacks (Miczek et al, 2013).

Molecular analysis of Bdnf expression. Genomic DNA was purified in parallel with total RNA from the same tissue punches. The tissues were homogenized in the lysis buffer, and each lysate sample was immediately divided into two portions and processed: one for RNA analysis by reverse-transcription real-time PCR and the other for DNA purification and methylation analysis by pyrosequencing (see detailed protocols in Supplementary Information).

Surgery and in vivo injection of TrkB drugs. Following the resident-intruder Test 1 , isolation-reared rats were submitted to either re-socialization alone (Re-socialization) or combined with fluoxetine (Re-socialization+fluoxetine). One week before the second resident-intruder test, the cannulas were bilaterally implanted to target the IL cortex. The activator of TrkB signaling 7,8-Dihydroxyflavone (DHF, $1 \mathrm{pM} /$ side), TrkB antagonist ANA-12 (0.1 pM/side), or vehicle (saline with $0.02 \%$ DMSO) were bilaterally microinjected in a volume of $100 \mathrm{nl} /$ side through the cannulas 26 , 14 , and $2 \mathrm{~h}$ before the second resident-intruder test (Test 2; see detailed protocols in Supplementary Information). The following groups of rats were used: 'Re-socialization +vehicle' $(N=7)$, 'Re-socialization+fluoxetine+vehicle' $(N=8)$, 'Re-socialization+DHF' $(N=7)$, and 'Re-socialization+fluoxetine+ANA-12' $(N=8)$. During the resident-intruder Test 2 , the main parameter of abnormal aggression, the share of vulnerable bites, was tested. Additional rats from 'Resocialization' $(N=6)$ and 'Re-socialization+fluoxetine' $(N=6)$ groups were not subjected to surgery and served as a control for the potential effect of surgery per se. Because the behavioral outcome of the groups not subjected to surgery and stereotaxically injected with vehicle were not significantly different, the groups were combined for statistical analysis and presentation on Figure 3 .

Connectivity analysis, $P V$, and PNN expression. Nanoinjections of the retrograde tracer cholera toxin $B$ subunit (CTb, Life Technologies, USA) were administered into the mPFC of rats deeply anesthetized by a mixture of ketamine, xylazine, and promethazinium chloratum. After 1 week, brains were sampled, cut into $60 \mu \mathrm{m}$ sections and $\mathrm{CTb}$ expression, and was visualized by immunohistochemical detection in brain regions where significant labeling occurred, and which have important roles in behaviorrelated prefrontal information processing, eg, the basolateral amygdala, hippocampus, and ventromedial and mediodorsal thalamus. The brains from the rats with a clear injection site of similar size between rats were analyzed. To correct for the inter-rat variability in the number of labeled cells, the input cell number in each region was normalized to the total input cell number. PV-PNN expression was investigated in the same brains by free-floating fluorescence immunohistochemistry. PV-PNN neurons were studied in the mPFC, hippocampus, and basolateral amygdala, ie, in brain regions that have a role either in the control of aggression or strongly influence prefrontal information processing and where both PV-containing interneurons and PNN were expressed (see detailed protocols in Supplementary Information).

\section{Statistical Analysis}

Values shown in text, table, and figures represent the mean \pm SEM. Main effects were studied by factorial or repeated-measures ANOVA as shown in text. When necessary, behavioral findings were square-root-transformed to fulfill ANOVA requirements. Post hoc analysis was made by the Duncan test unless otherwise stated. The regression analysis was done using a StatSoft 12.0 software. The comparison between the treatment groups and a socially reared group was done by two-tailed unpaired $t$-test. $P$-values lower than 0.05 were considered statistically significant.

\section{RESULTS}

\section{Post-Weaning Social Isolation Induces Abnormal Forms of Aggression in the Resident-Intruder Test}

To study the impact of early social isolation on aggression, we performed a first resident-intruder test (Test 1). In line with earlier observations, the 8-week-long post-weaning social isolation led to escalated aggression as compared to socially reared rats. Particularly, rats reared in social isolation delivered more bites $(\mathrm{F}(1,121)=25.21 ; p<0.0001)$ and targeted more bites toward vulnerable body parts of opponents (head, throat, belly; $\mathrm{F}(1,121)=12.19 ; p<0.001$; Figure 1a). Moreover, the number of bites delivered shifted from less to more damaging hard bites in isolation-reared rats as compared to socially reared rats (interaction rearing $x$ bite type; $\mathrm{F}(2,242)=4.23 ; p<0.05$; Figure 1a, right-hand panel; see Supplementary Information for the description of attack types).

\section{Post-Weaning Social Isolation-Reared Rats are Capable of Social Learning}

Subsequent to the resident-intruder Test 1, rats reared in social isolation were either housed individually or were introduced into social groups for 3 weeks (re-socialization), and the groups were further subdivided according to fluoxetine treatment. During the first hour of re-socialization of isolation-reared rats and re-grouping of socially reared rats, bite counts and offensive threats were similar (Supplementary Figure S1). In the same period, assertive 
a
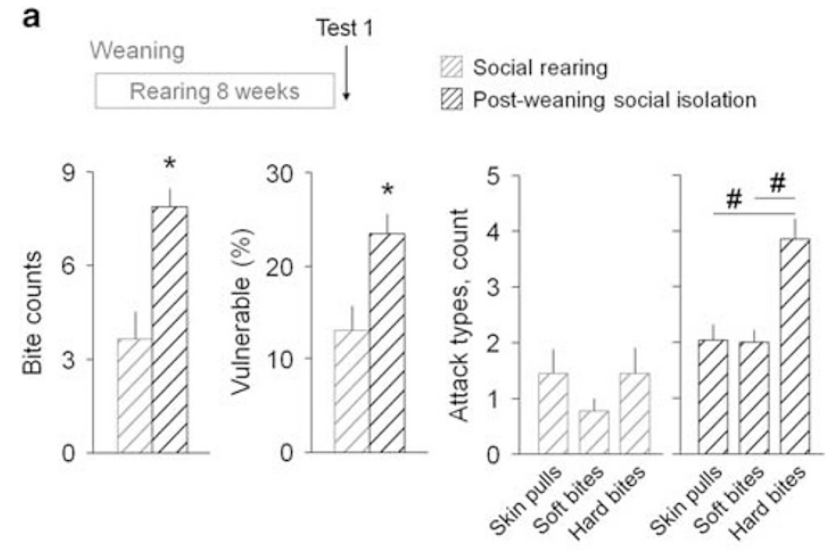

b
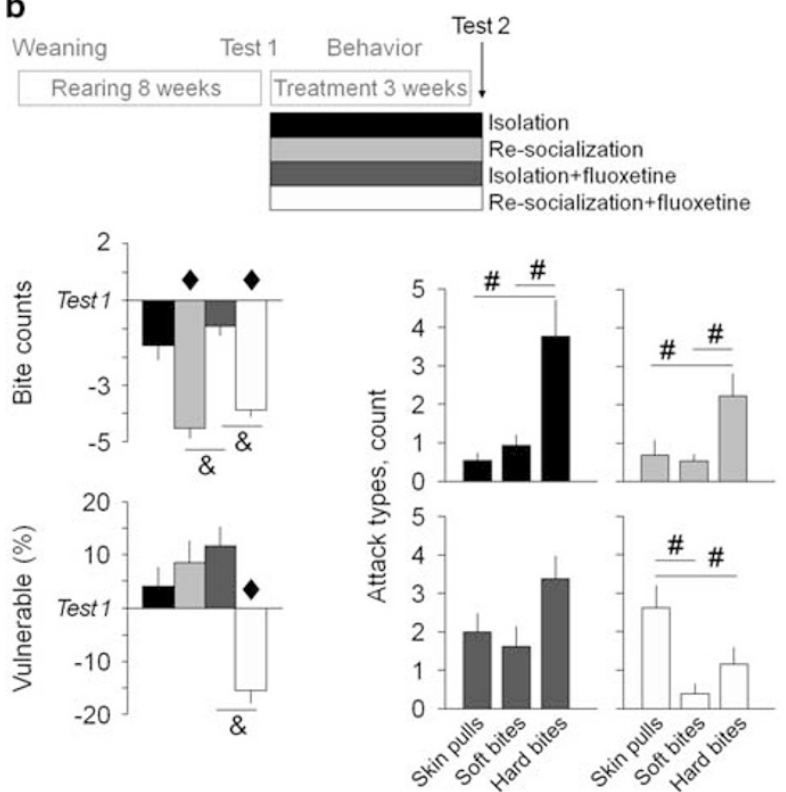

Figure I Behavioral effects of post-weaning social isolation and resocialization with or without fluoxetine treatment. (a) In the first residentintruder test (Test I), isolation-reared rats bit opponents more frequently, and delivered more bites to vulnerable body parts of opponents (head, throat, and belly). Hard bites were more dominant over soft bites and skin pulls in isolation-reared rats as compared to socially reared rats. $N=40-80$ rats/group. (b) In the second resident-intruder Test 2, the combined treatment reduced the number of bites delivered, and the share of bites aimed at vulnerable targets as compared with the Test I. The dominance of hard bites over soft bites and skin pulls was reversed by the combined treatment Re-socialization+fluoxetine. More minor effects were observed with single treatments. $N=12-13$ rats/group. Data are present as mean \pm SEM. ${ }^{*} p<0.05$ between socially reared and isolation-reared groups; ${ }^{\#} p<0.05$ between the attack types; $p<0.05$ between the residentintruder Test I and Test $2 ;{ }^{\&} p<0.05$ between the treatment groups.

aspects of social behavior decreased in isolation-reared rats, as shown by decreased propensity to show dominant posture, and increased unjustified defense responses, ie, defensive behaviors that occur in response to non-offensive actions of opponents. These social deficits were abolished over the first week of cohabitation, when an overall decrease in aggression was observed in all groups as compared to the first hour of social cohabitation (Supplementary Figure S1). An analogous pattern of changes was observed in social cohesion as measured by huddling during sleep (Supplementary Figure S1). Huddling was very low in isolationreared rats on the first day, but gradually increased, and the difference between socially reared and isolation-reared groups disappeared after 1 week. The temporal pattern of huddling behavior showed a learning curve-like behavioral change, supporting the view that social learning took place, albeit a habituation-induced reduction of emotional responses can increase social interaction as well. Fluoxetine did not have significant influence on the effects of social cohabitation (Supplementary Figure S1).

Thus, post-weaning social isolation resulted in social deficits, which were, however, only transient, and were readily abolished by social cohabitation, demonstrating that social learning did take place.

\section{Social Learning Restores Post-Weaning Social Isolation- Induced Abnormal Aggression only When Combined with Fluoxetine}

To investigate the impact of treatments on aggressiveness, rats reared in social isolation were submitted to a second resident-intruder test (Test 2). Treatments did affect aggression as compared to the first resident-intruder test (Test 1 ; bite counts: $\mathrm{F}(4,90)=4.26 ; p<0.01$; vulnerable bites: $\mathrm{F}(4,90)=3.13 ; p<0.02)$, but their efficacies were markedly different (Figure 1b). Re-socialization alone decreased the bite counts (repeated-measure ANOVA, interaction Test $\times$ re-socialization: $\mathrm{F}(1,48)=9.18 ; p<0.01$ ), while the share of vulnerable bites was affected only in the group received the combined treatment (interaction fluoxetine $\times$ re-socialization: $\mathrm{F}(1,48)=7.98 ; p<0.01)$. Fluoxetine alone affected neither form of abnormal aggressive behavior investigated here. The combined treatment, thus, ameliorated all three measures of aggressiveness: bite counts $(4.2 \pm 0.7)$ and the share of vulnerable bites $(14.1 \pm 5.5 \%)$ were restored to the original levels of socially reared rats (shown on Figure 1a; $p>0.05$ for both parameters, two-tailed unpaired $t$-test), whereas the dominance of hard bites over skin pulls was reversed (Figure 1b). This is in contrast to the effect of fluoxetine in socially reared and regrouped rats that lacked the social learning component of re-socialization, which was present in isolation-reared rats that had no social experience earlier; fluoxetine decreased bite counts $(\mathrm{F}(1,23)=4.91 ; p<0.05)$, but did not affect the share of vulnerable attacks $(\mathrm{F}(1,23)=$ 1.28; $p>0.05$; Supplementary Figure S2).

\section{The Combined Treatment of Post-Weaning Socially Isolated Rats Targets Bdnf Signaling in the IL Cortex}

Post-weaning social isolation represses and the combined treatment restores Bdnf expression. Previous studies showed that behavioral effects of fluoxetine on neuronal plasticity are dependent of Bdnf-TrkB signaling (Castren and Rantamaki, 2010; Karpova et al, 2011). To investigate the impact of post-weaning social isolation and treatments on Bdnf, we studied the expression of Bdnf transcripts 1 and 4 in the aggression neuronal circuitry (Supplementary Figure S3) at the end of treatment period. In the absence of treatment ('Isolation' group, Figure 2a), post-weaning social isolation drastically reduced Bdnf levels in the IL cortex and MeA (both transcripts), and LH (Bdnf1; Figure 2b). 
a
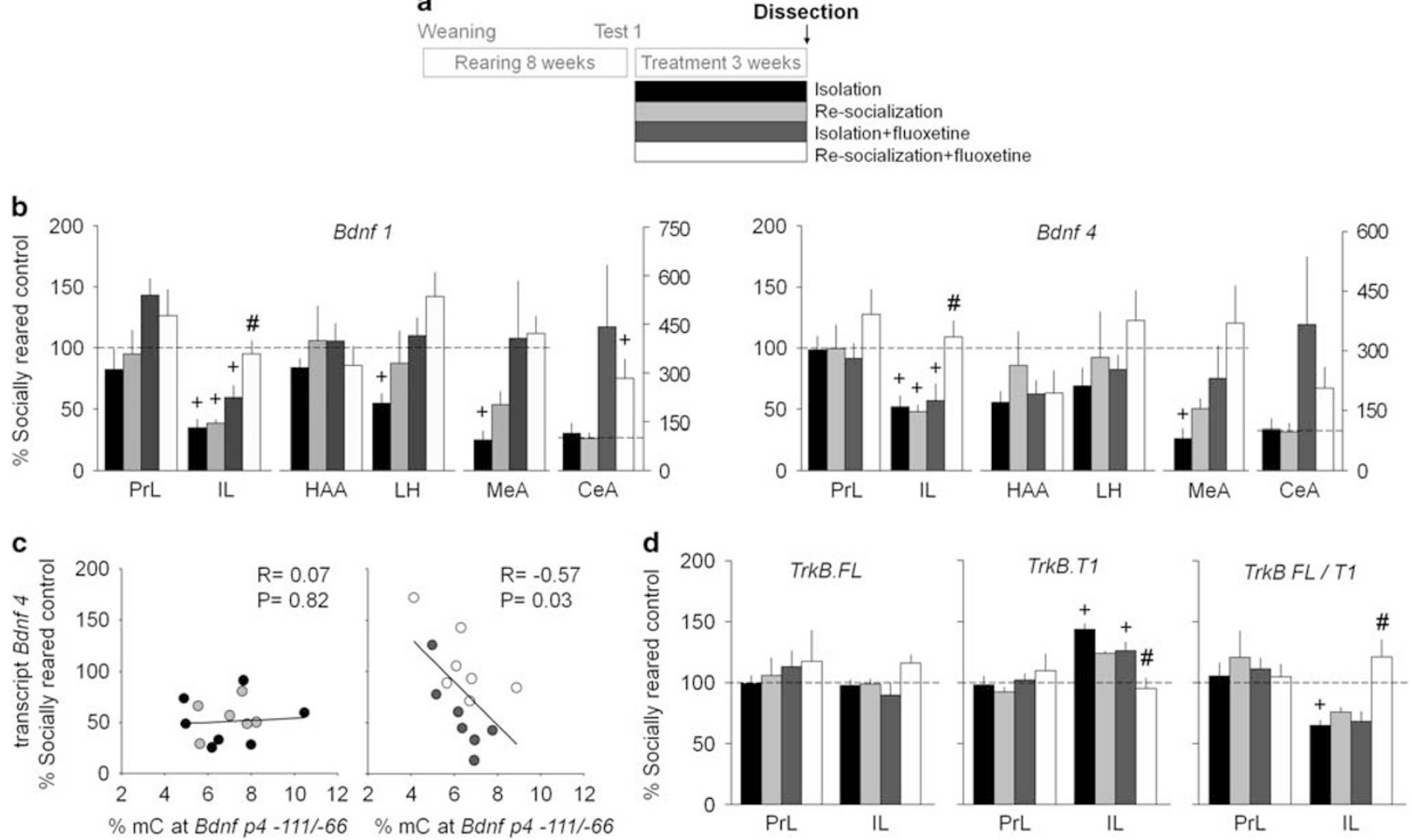

Figure 2 Combined treatment alters Bdnf and TrkB expression in the infralimbic cortex of isolation-reared rats. (a) The experimental protocol for tissue dissection. (b) Post-weaning social isolation reduced the expression of both BDNF variants in the infralimbic (IL) but not in the prelimbic (PrL) cortex. The effect was abolished specifically by the combined treatment. The rearing in isolation also decreased Bdnf I in the lateral hypothalamus (LH), as well as Bdnf I and 4 levels in the medial amygdala (MeA). Fluoxetine upregulated Bdnf I and 4 levels independently of re-socialization (see the text) in the LH, MeA, and central amygdala (CeA). HAA, hypothalamic attack area. (c) Fluoxetine treatment induced a significant correlation between Bdnf 4 levels and the \% methylated cytosine (mC) at Bdnf p4- I I / /-66 in the IL. (d) In the infralimbic cortex, post-weaning social isolation induced the expression of the dominant-negative isoform TrkB.TI of Bdnf receptor TrkB, while the combined treatment restored the levels of TrkB.TI and the ratio TrkB.FL/TI to the levels found in socially reared rats. Data are present as mean \pm SEM. ${ }^{+} p<0.05$ between the treatment and socially reared control groups (two-tailed $t$-test); ${ }^{*} p<0.05$ compared to the Isolation treatment group (post hoc analysis). $N=6-7$ rats/group.

Although fluoxetine upregulated Bdnf in the PrL cortex $(B d n f 1 \mathrm{~F}(1,23)=6.53, p<0.05), \mathrm{MeA}(B d n f 1 \mathrm{~F}(1,22)=5.06$, $p<0.05 ; B d n f 4 \mathrm{~F}(1,22)=6.77, p<0.05)$, LH $(B d n f 1 \mathrm{~F}(1,23)=$ 9.63, $p<0.01)$, and CeA $(B d n f 1 \mathrm{~F}(1,22)=5.44, p<0.05)$ of isolation-reared rats, this effect was independent of re-socialization $(p>0.05)$. These findings suggest that fluoxetine might affect neuronal plasticity in these regions but the changes observed had little impact on this behavior, as fluoxetine alone did not affect aggression. By contrast, both treatments together upregulated Bdnf transcripts 1 and 4 in the IL region of the MPFC (Bdnf1 re-socialization $\mathrm{F}(1,23)=5.01, p<0.05$, fluoxetine $\mathrm{F}(1,23)=22.36, p<0.0001$; Bdnf4 re-socialization $\mathrm{F}(1,23)=4.24, \quad p=0.05$, fluoxetine $\mathrm{F}(1,23)=8.29, p<0.01$; Figure $2 \mathrm{~b})$. More specifically, the Bdnf4 expression was controlled by significant interaction of the re-socialization and fluoxetine factors $(\mathrm{F}(1,23)=5.78$, $p<0.05)$. Expression of Bdnf transcripts 1 and 4 was not affected by fluoxetine treatment in socially reared rats (Supplementary Figure S4).

Fluoxetine shows epigenetic regulation of Bdnft expression. Because DNA methylation at the activity-dependent Bdnf promoter p4 was consistently reported as a repressive regulatory mechanism for Bdnf4 transcription (Lubin et al, 2008; Onishchenko et al, 2008), we investigated DNA methylation levels at Bdnf p4 in the IL cortex. Post-weaning social isolation did not influence the methylation of $\mathrm{CpG}$ island of the p4 promoter. However, we observed a significant negative correlation between Bdnf4 mRNA levels and $\mathrm{p} 4$ methylation in the fluoxetine-treated groups, but not in the vehicle groups (Figure 2c). This suggests that individual fluoxetine-induced changes in promoter methylation are a permissive factor that potentiates the effects of re-socialization on Bdnf expression in the IL cortex.

TrkB-signaling in the IL cortex mediates the effect of combined treatment in rats reared in social isolation. As fluoxetine treatment combined with re-socialization particularly affected Bdnf expression in the IL cortex, we investigated whether signaling through the Bdnf receptor TrkB has a functional role in the rescuing effect of the combined treatment. First, we analyzed whether the TrkB expression in the mPFC was also altered by post-weaning social isolation or treatments. In the PrL cortex, neither postweaning social isolation, nor treatments altered the levels of the full-length TrkB.FL transcript and its major truncated 

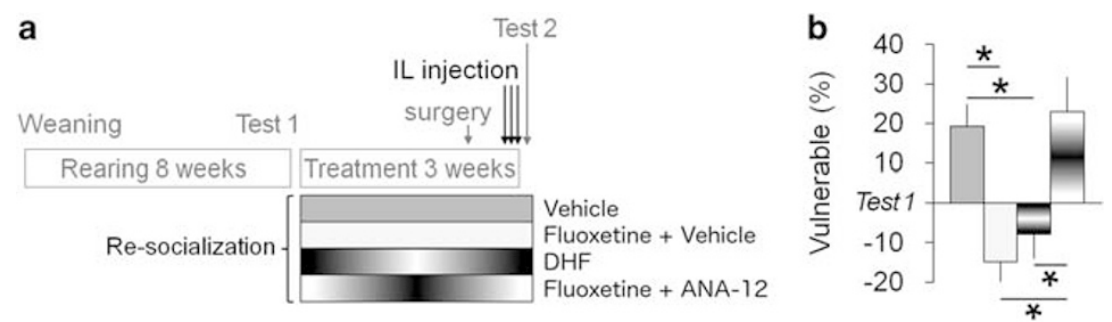

Figure 3 TrkB signaling in the infralimbic cortex mediates the effect of combined treatment on abnormal aggression. (a) The experimental protocol used to analyze the role of TrkB signaling in the combined treatment outcome. IL, infralimbic cortex. (b) The activation of TrkB signaling in the IL cortex by 7,8Dihydroxyflavone (DHF) mimicked the effect of fluoxetine on the share of vulnerable bites when combined with re-socialization, whereas the effect of the combined treatment Re-socialization+fluoxetine was inhibited by the TrkB antagonist ANA-12. Data are present as mean \pm SEM. * $p<0.05$ between the groups (post hoc analysis). $N=7-14$ rats/group.

isoform TrkB.T1 ( $p>0.05$; Figure 2d). In contrast, in the IL cortex, post-weaning social isolation upregulated TrkB.T1 levels and, thus, reduced the ratio TrkB.FL/T1 (Figure 2d). Only the combined treatment restored these parameters to the levels found in socially reared rats; specifically, the TrkB. FL/T1 ratio was controlled by the interaction of the resocialization and fluoxetine factors $(\mathrm{F}(1,20)=5.85, p<0.05)$.

Because TrkB.T1 isoform can dimerize with the full-length TrkB receptor to inhibit its activation (Saarelainen et al, 2000), we hypothesized that the beneficial effect of fluoxetine in rats reared in isolation and then re-socialized may implicate the activation of TrkB receptor. We stereotaxically targeted the IL cortex (Supplementary Figure S5) of (1) rats received re-socialization treatment with the activator of TrkB signaling DHF, and (2) rats received the combined treatment with the small-molecule TrkB antagonist, ANA-12, at the end of treatment (Figure 3). Next, we analyzed the critical parameter of abnormal aggression, the share of vulnerable attacks, in the second resident-intruder test Test 2. DHF mimicked the effect of fluoxetine in the combined treatment, whereas the inhibition of TrkB signaling by ANA-12 completely abolished the effect of the combined treatment on post-weaning social isolation-induced abnormal aggression (Figure $3 \mathrm{~b}$ ). In addition, the level of aggressiveness showed a pattern toward reduction in both Re-socialization+DHF and Re-socialization+fluoxetine+ANA-12 groups (bite count $-14.43 \pm 8.87, p>0.1$, and $-7.38 \pm 5.77, p>0.1$, respectively; hard bites $-8.57 \pm 4.23, p=0.09$, and $-5.00 \pm 2.74, p>0.1$, respectively; the comparison between the resident-intruder Tests 1 and 2 by the two-tailed paired $t$-test).

\section{Combined Treatment Induces Structural Remodeling of Aggression-Specific Network}

Following the resident-intruder Test 2, the retrograde tracer Cholera toxin $\beta$ was injected into the mPFC of rats (Figure $4 \mathrm{a}$ and b). Projection intensities and the density of PV interneurons surrounded by PNN, a marker of neuronal plasticity (Nowicka et al, 2009), were investigated after 1 -week incubation. Out of the four major brain regions projecting to the $\mathrm{mPFC}$ from the limbic system, the combined treatment specifically increased the density of retrogradely labeled neurons in the CA1 area of the ventral hippocampus (interaction of the re-socialization and fluoxetine factors $\mathrm{F}(1,20)=5.54, p<0.05$; Figure $4 \mathrm{c}-\mathrm{f}$ and Supplementary Figure S6). The number of PV-PNN neurons also decreased specifically in the vHIP, consistent with the reopening of plastic changes in this brain area (Figure 4e and f). Although fluoxetine reduced PV-PNN both alone and in combination with re-socialization (ANOVA effect of fluoxetine $\mathrm{F}(1,20)=7.10, p<0.05)$, multiple regression analysis suggested that this fluoxetine-induced plasticity significantly contributed to the behavioral improvement only in the group receiving the combined treatment. Particularly, the share of vulnerable attacks interactively correlated with vHIP-mPFC projections and PV-PNN labeling in the vHIP (Multiple $R=0.569 ; \mathrm{F}(2,31)=4.15 ; p<0.05$ ). Moreover, the two neuroanatomical variables explained a substantial share of behavioral variation (adjusted $R^{2}=0.324$; variance explained: 32.4\%; Figure 3d). By contrast, PV-PNN labeling in the vHIP did not correlate with vulnerable attacks $(R=0.283 ; \mathrm{F}(2,17)=1.66 ; p>0.05)$, whereas vHIP-mPFC projections did show a significant correlation $(R=0.421$; $\mathrm{F}(2,17)=4.74 ; \quad p<0.05)$, but the behavioral variation explained was substantially smaller (adjusted $R^{2}=0.177$; variance explained: 17.7\%). The large share of behavioral variation explained by the two neuroanatomical changes observed in the ventral hippocampus suggest that this brain area is part of the mechanisms by which the combined treatment ameliorated aggression induced by post-weaning social isolation.

Taken together, these findings show that, similar to changes in aggression, neural plasticity was affected by an interaction between psychosocial and pharmacological treatments in the mPFC, an area of key importance in the top-down control of subcortical mechanisms of aggression (Siever, 2008).

\section{DISCUSSION}

It was recently suggested that in the case of aggression problems, socially acceptable treatments should use pharmacological tools to enhance the effects of positive social experiences rather than to suppress symptoms (Dadds and Rhodes, 2008). Here we show that the combination of social learning and fluoxetine, but neither treatment per se, abolishes abnormal aggression resulting from adverse early experiences. It appears that changes induced in the mPFC, particularly the IL, are relevant in this respect. Our findings in the amygdala and hypothalamus, which also have important roles in the control of aggression (Siegel et al, 2007), show that fluoxetine affects neuronal plasticity in these regions as well; however, this had little impact on 
a

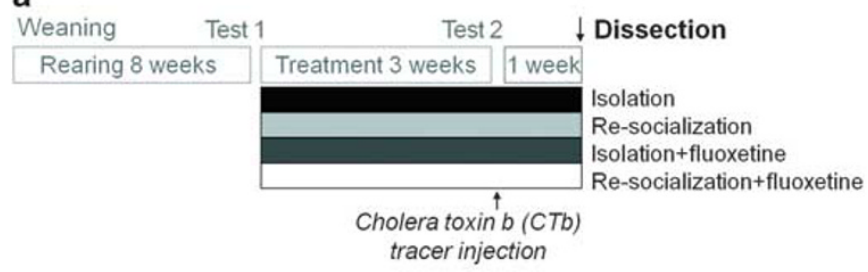

c

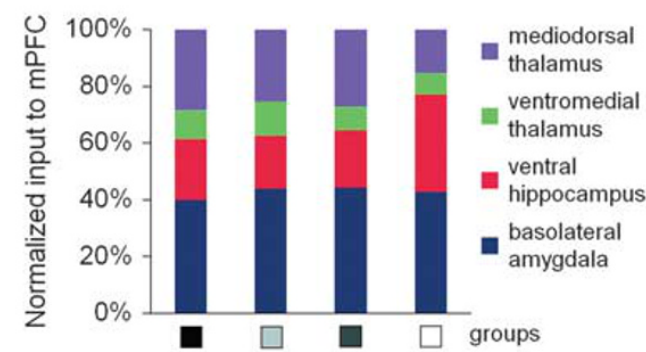

d

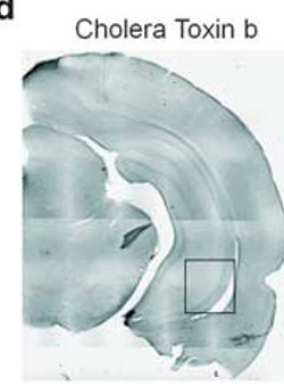

e

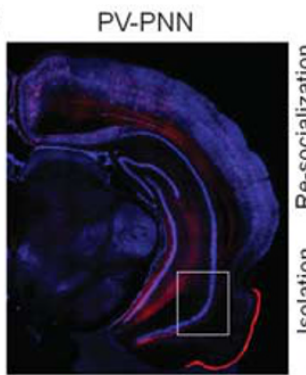

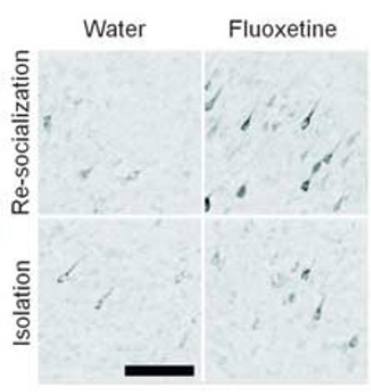

Water

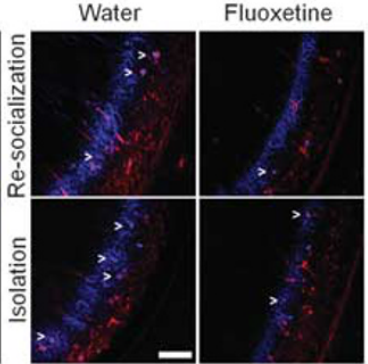

b

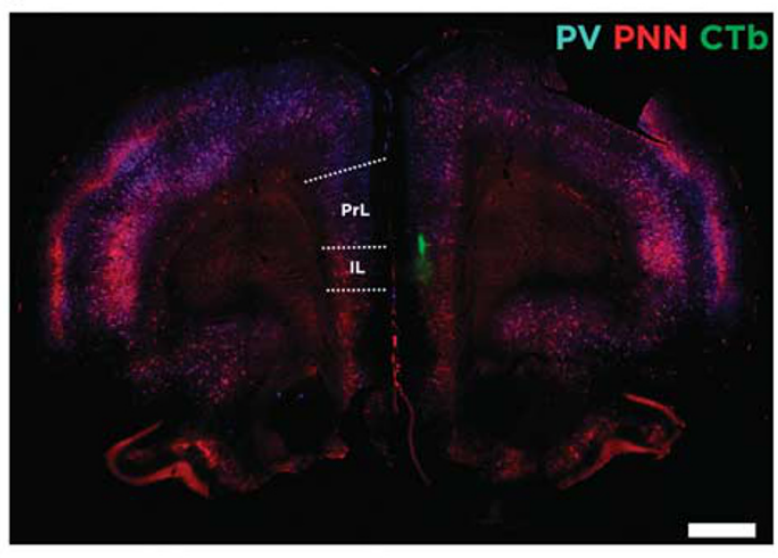

f
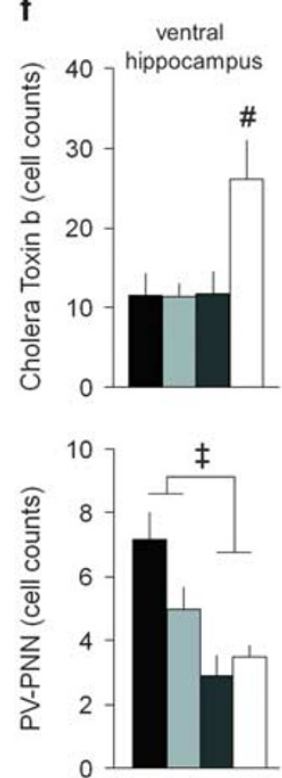

g

Multiple $\mathrm{R}=0.569$

$F(2,31)=4.15 ; p=0.02$

adjusted $R^{2}=0.324$

Variance explained: $32.4 \%$

Figure 4 Network remodeling in the ventral hippocampus (VHIP). (a) The experimental protocol. (b) Confocal tile image showing the injection site at the border between prelimbic (PrL) and infralimbic (IL) cortices, in the medial prefrontal cortex (mPFC). Scale bar = I mm. (c) Histogram showing the normalized input intensity arriving to the mPFC from all the analyzed projecting areas to correct intersubject variability. The relative weight of the ventral hippocampus (vHIP) projections in the group Re-socialization+fluoxetine is significantly increased compared to any other group (the multiple $t$-test comparison to the groups: Isolation $p=0.039$, Re-socialization $p=0.015$, and Isolation+fluoxetine $p=0.027$ ). (d) Photomicrograph tile image showing the vHIP neurons labeled with cholera toxin b (CTb). (e) Confocal tile scan image showing the expression of parvalbumin (PV, blue) and perineuronal nets (PNN, red). (d, e) Left: the squared region shows the area studied (the CAI stratum of the vHIP). Right: representative images showing labeled neurons in experimental groups. Scale bars $=100 \mathrm{\mu m}$. (f) The density of CTb labeling and neurons double-labeled for PV and PNN in the vHIP I week after the injection of the tracer into the mPFC. Data are present as mean \pm SEM. ${ }^{\ddagger} p<0.05$ between fluoxetine treated and non-treated groups; ${ }^{\#} p<0.05$ compared to the Isolation-treated group (post hoc analysis). (g) 3D plot of the interaction between CTb labeling, PV-PNN cells in the vHIP, and the \% vulnerable hard bites. $N=5-7$ rats/group.

abnormal aggression, as fluoxetine alone did not affect this behavior. Our epigenetic findings, together with Bdnf-TrkB signaling, connectivity and PV-PNN data suggest that fluoxetine exerted its neuronal plasticity-enhancing effects through molecular and cellular alteration in the mPFC network.

\section{The Effect of Re-Socialization on Post-Weaning Social Isolation-Induced Abnormal Aggression}

Re-socialization may intuitively occur as an appropriate treatment of social deprivation; however, it failed to correct post-weaning social isolation-induced deficits in a number of behavioral paradigms including aggression, drug addiction, and social learning (Baarendse et al, 2014; Kercmar et al, 2011; Tulogdi et al, 2014). A similar phenomenon was observed in humans, where social learning through psychotherapy modestly ameliorated violent behavior that frequently results from early adversities (Currie and Startup, 2012; Weiss et al, 2005). Consistently, and in line with our Bdnf expression data, brain alterations induced by post-weaning social isolation, such as the ventral tegmental area synaptic properties, persist fully or partially when isolation rearing is followed by a period of 
re-socialization (Baarendse et al, 2013; Bledsoe et al, 2011; Pascual et al, 2006; Whitaker et al, 2013). We suggest here that re-socialization was ineffective alone because neural plasticity was low in adult rats and social learning was unable to durably affect brain mechanisms underlying deviant forms of aggression. The expression of plasticity-related genes Bdnf and TrkB appears to be at least partially responsible for the observed social impairment. Particularly, post-weaning social isolation-induced changes in Bdnf and TrkB.FL/T1 expression profiles in the IL cortex were ameliorated by the combined treatment, whereas re-socialization had no effect alone. Indeed, activation of TrkB signaling by DHF mimicked the effects of fluoxetine, while TrkB inhibitor blocked the reduced aggression produced by combined fluoxetine and re-socialization in isolation-reared rats, suggesting a causal role of $\operatorname{TrkB}$ signaling in this process. However, social behavior in newly formed groups was normalized by re-socialization alone. As such, alterations in Bdnf or TrkB expression did not seem to affect social behavior in groups, but seemed to be linked to aggressiveness.

\section{The Effect of Fluoxetine on Post-Weaning Social Isolation-Induced Abnormal Aggression}

The inefficacy of fluoxetine alone in isolation-reared rats may be surprising, as fluoxetine treatment has been connected with reduced aggressiveness (Fuller, 1996). In line with these earlier findings, fluoxetine reduced aggression in socially reared rats, albeit modestly. However, a meta-analysis revealed that its effects on rodent aggression depend on a variety of factors; particularly weak effects were obtained in Wistar rats submitted to social isolation (Carrillo et al, 2009). Fluoxetine failed to ameliorate aggressiveness in an intermittent explosive disorder (Coccaro et al, 2015), which often results from early social neglect, and for which post-weaning social isolation may serve as a model (Haller et al, 2014; Toth et al, 2011). In line with behavioral inefficacy, fluoxetine alone failed to restore Bdnf signaling deficits in the $\mathrm{mPFC}$ - the highest-order controlling structure of aggression (Siever, 2008)-and also failed to influence mPFC connectivity that appear necessary for the reduction in aggression as shown by Multiple Regression analysis, albeit it affected aspects of neuronal plasticity in other brain regions.

\section{Fluoxetine and Bdnf Promoter Methylation}

In studies investigating monocular deprivation (Maya Vetencourt et al, 2008) and learned fear extinction (Karpova et al, 2011), the favorable role of fluoxetine was attributed to its effects on various aspects of neuronal plasticity including Bdnf gene expression profiles and PV-PNN expression. Our DNA methylation analysis provides novel insight into the plasticity-related effects of fluoxetine. We suggest that chronic fluoxetine enhanced Bdnf4 transcription via decreased DNA methylation at the Bdnf promoter $\mathrm{p} 4$. The epigenetic mechanisms of Bdnf expression, and especially Bdnf4 exon, have widely implicated the histone-modifying machinery as a key regulator of antidepressant-dependent Bdnf transcription (Karpova, 2014). However, so far, the role of DNA methylation in antidepressant effects was poorly investigated. Two studies reported that fluoxetine treatment, either acute (Sales and Joca, 2016) or chronic during pregnancy and lactation (Toffoli et al, 2014), resulted in decreased global DNA methylation, although no correlation analysis between the levels of DNA methylation and gene expression in the same tissue sample was performed. Furthermore, different environmental events may target distinct $\mathrm{CpG}$ sites at gene promoters. The 6 weeks of chronic ultramild stress exposure especially induced CpG site \#2 near the CREB-binding site at the promoter for the glial cell-derived neurotrophic factor Gdnf, rendering Gdnf expression dependent on CREB function (Uchida et al, 2011). The methylation level at the CpG-87, within the PasRE element in Bdnf p4 island that we studied here, could serve as predictive marker for antidepressant response in depressive patients (Tadic et al, 2014), although the molecular mechanism of that observation is not known. The PasRE element is a binding site for Npas4 factor critically involved in fluoxetine-induced neuronal plasticity (Maya-Vetencourt et al, 2012) and Bdnf control (Pruunsild et al, 2011). A 4-week-long post-weaning social isolation in mice downregulates Npas4 expression (Ibi et al, 2008). Moreover, the Npas4 deficiency specifically during adolescence impairs the normal maturation pattern of prefrontal inhibitory circuits by regulating the parvalbumin neurons and the activity-dependent Bdnf expression in mice (Shepard et al, 2017). It is thus reasonable to suggest that fluoxetine, via an Npas4-binding site methylation, might act at rescuing the developmentally induced impairment of the mPFC inhibitory circuits' maturation. Further studies should investigate the potential role of Npas4 in the development of abnormal aggression.

\section{The Role of the mPFC in the Control of Escalated Aggression}

The mPFC is a critical site of aggression control (Siegel et al, 2007), and several lines of evidence suggest that dysfunctions in this area promote unrestricted-abnormal-aggression in both animals and humans (Blair, 2015; Marquez et al, 2013; Takahashi et al, 2014). Recent evidence from other areas of behavioral research suggests that the general role of $\mathrm{mPFC}$ can be broken down into separate roles played by its subregions. For instance, top-down control of anxiety is exerted by the IL cortex but not by the PrL cortex (Adhikari et al, 2015). Our findings on Bdnf-TrkB signaling suggest a similarly distinct role for the IL cortex in the ameliorative effects of the combined treatment. Previous findings implicate BDNF production in the MPFC and hippocampus in aggression-related behavior (Maynard et al, 2016), but the hippocampal connections to the mPFC have received little attention so far. The ventral hippocampus shows robust neural activation during emotionally salient challenges (Beck and Fibiger, 1995) and it is highly connected to the limbic system with robust inputs to the prefrontal cortex (Jay and Witter, 1991). Moreover, an in vivo electrophysiological study revealed that the ventral-hippocampal and prefrontocortical theta oscillations were highly correlated (Adhikari et al, 2011). It is intriguing to examine whether hippocampal projections to the IL and PrL cortices have a distinct role in the regulation of aggressive behaviors. 
We suggest that the enhancement of hippocampal projection to the $\mathrm{mPFC}$ can disrupt aggressive behaviors in a similar manner as it disrupts the processing of fear expression (SotresBayon et al, 2012): through competition for target innervation, which is dependent on Bdnf expression in the IL cortex (Rosas-Vidal et al, 2014). This is supported by recent findings that show that the ventral hippocampus conveys spatial information to the mPFC (Spellman et al, 2015), where it would compete against other limbic inputs such as the basolateral amygdala (Senn et al, 2014). During the critical period, these different inputs would compete for target innervation resulting in a specific long-lasting $\mathrm{mPFC}$ network configuration (Guirado et al, 2016). During adulthood, in the absence of high levels of plasticity, we hypothesize that changes in the intensity of their inputs would only produce transient behavioral changes-not only related to aggression, but also to fear expression or depressive-related behavior. In this line, optogenetic and chemogenetic stimulation of this projection during adulthood is sufficient to produce a rapid antidepressant-like response (Carreno et al, 2016).

\section{Perspectives}

Escalated human aggression, which is believed to result from early adverse experiences (Fanning et al, 2014), shows multiple similarities with the behavior of isolation-reared rats (Haller and Kruk, 2006). In addition to those reviewed earlier, present findings reveal further similarities between the model and the modeled phenomenon. Although aggressiveness markedly increased in isolation-reared rats when they were challenged by an intruder in their home-cage, the very same rats were rather timid when they encountered several partners in an unfamiliar arena, suggesting the emergence of a social anxiety-like response. This mirrors the human case, where emotional aggression is also associated with social deficits and is characterized by a contextdependent expression of both aggression and anxiety (Kessler et al, 2006; Keyes et al, 2016). On the basis of multiple similarities between the behavior of isolation-reared rats and developmentally induced aggression in humans, and considering that re-socialization models important aspects of behavioral therapy (Miczek et al, 2013), we suggest that re-opening neuronal plasticity by fluoxetine can increase the efficacy of psychotherapeutic interventions in developmentally induced aggression problems.

\section{FUNDING AND DISCLOSURE}

This study was supported by the grant CAPES (Brazil) to NNK; ERC grant No 322742-iPLASTICITY, Sigrid Juselius foundation and Academy of Finland grant \#257486 to EC; and NKFI grant No. 112907 to JH. The authors declare no conflict of interest.

\section{ACKNOWLEDGMENTS}

We thank Drs Tomi Rantamäki (UH, Helsinki) and Sãmia Joca (FCFRP, Ribeirao Preto) for valuable comments, and Andrea Miro-Canturri, Outi Nikkilä, Sulo Kolehmainen, and Beata Barsvari for technical help.

\section{REFERENCES}

Adhikari A, Lerner TN, Finkelstein J, Pak S, Jennings JH, Davidson TJ et al (2015). Basomedial amygdala mediates top-down control of anxiety and fear. Nature 527: 179-185.

Adhikari A, Topiwala MA, Gordon JA (2011). Single units in the medial prefrontal cortex with anxiety-related firing patterns are preferentially influenced by ventral hippocampal activity. Neuron 71: 898-910.

Baarendse PJ, Counotte DS, O'Donnell P, Vanderschuren LJ (2013). Early social experience is critical for the development of cognitive control and dopamine modulation of prefrontal cortex function. Neuropsychopharmacology 38: 1485-1494.

Baarendse PJ, Limpens JH, Vanderschuren LJ (2014). Disrupted social development enhances the motivation for cocaine in rats. Psychopharmacology 231: 1695-1704.

Bavelier D, Levi DM, Li RW, Dan Y, Hensch TK (2010). Removing brakes on adult brain plasticity: from molecular to behavioral interventions. J Neurosci 30: 14964-14971.

Beck CH, Fibiger HC (1995). Conditioned fear-induced changes in behavior and in the expression of the immediate early gene c-fos: with and without diazepam pretreatment. J Neurosci 15: 709-720.

Blair RJ (2015). Psychopathic traits from an RDoC perspective. Curr Opin Neurobiol 30: 79-84.

Bledsoe AC, Oliver KM, Scholl JL, Forster GL (2011). Anxiety states induced by post-weaning social isolation are mediated by CRF receptors in the dorsal raphe nucleus. Brain Res Bull 85: 117-122.

Carreno FR, Donegan JJ, Boley AM, Shah A, DeGuzman M, Frazer A et al (2016). Activation of a ventral hippocampus-medial prefrontal cortex pathway is both necessary and sufficient for an antidepressant response to ketamine. Mol Psychiatry 21: 1298-1308.

Carrillo M, Ricci LA, Coppersmith GA, Melloni RH Jr (2009). The effect of increased serotonergic neurotransmission on aggression: a critical meta-analytical review of preclinical studies. Psychopharmacology 205: 349-368.

Castren E, Rantamaki T (2010). The role of BDNF and its receptors in depression and antidepressant drug action: Reactivation of developmental plasticity. Dev Neurobiol 70: 289-297.

Coccaro EF, Lee R, Breen EC, Irwin MR (2015). Inflammatory markers and chronic exposure to fluoxetine, divalproex, and placebo in intermittent explosive disorder. Psychiatry Res 229: 844-849.

Coccaro EF, Lee RJ, Kavoussi RJ (2009). A double-blind, randomized, placebo-controlled trial of fluoxetine in patients with intermittent explosive disorder. J Clin Psychiatry 70: 653-662.

Currie M, Startup M (2012). Doing Anger Differently: two controlled trials of percussion group psychotherapy for adolescent reactive aggression. $J$ Adolesc 35: 843-853.

Dadds MR, Rhodes T (2008). Aggression in young children with concurrent callous-unemotional traits: can the neurosciences inform progress and innovation in treatment approaches? Philos Trans $R$ Soc Lond B Biol Sci 363: 2567-2576.

Fanning JR, Meyerhoff JJ, Lee R, Coccaro EF (2014). History of childhood maltreatment in intermittent explosive disorder and suicidal behavior. J Psychiatr Res 56: 10-17.

Fuller RW (1996). The influence of fluoxetine on aggressive behavior. Neuropsychopharmacology 14: 77-81.

Gilbert R, Widom CS, Browne K, Fergusson D, Webb E, Janson S (2009). Burden and consequences of child maltreatment in highincome countries. Lancet 373: 68-81.

Guirado R, Perez-Rando M, Sanchez-Matarredona D, Castren E, Nacher J (2014). Chronic fluoxetine treatment alters the structure, connectivity and plasticity of cortical interneurons. Int $J$ Neuropsychopharmacol 17: 1635-1646.

Guirado R, Umemori J, Sipila P, Castren E (2016). Evidence for competition for target innervation in the medial prefrontal cortex. Cereb Cortex 26: 1287-1294. 
Haller J, Harold G, Sandi C, Neumann ID (2014). Effects of adverse early-life events on aggression and anti-social behaviours in animals and humans. J Neuroendocrinol 26: 724-738.

Haller J, Kruk MR (2006). Normal and abnormal aggression: human disorders and novel laboratory models. Neurosci Biobehav Rev 30: 292-303.

Hubel DH, Wiesel TN, LeVay S (1977). Plasticity of ocular dominance columns in monkey striate cortex. Philos Trans $R$ Soc Lond B Biol Sci 278: 377-409.

Ibi D, Takuma K, Koike H, Mizoguchi H, Tsuritani K, Kuwahara Y et al (2008). Social isolation rearing-induced impairment of the hippocampal neurogenesis is associated with deficits in spatial memory and emotion-related behaviors in juvenile mice. J Neurochem 105: 921-932.

Jay TM, Witter MP (1991). Distribution of hippocampal CA1 and subicular efferents in the prefrontal cortex of the rat studied by means of anterograde transport of Phaseolus vulgarisleucoagglutinin. J Comp Neurol 313: 574-586.

Karpova NN (2014). Role of BDNF epigenetics in activitydependent neuronal plasticity. Neuropharmacology 76(Pt C): 709-718.

Karpova NN, Pickenhagen A, Lindholm J, Tiraboschi E, Kulesskaya N, Agustsdottir A et al (2011). Fear erasure in mice requires synergy between antidepressant drugs and extinction training. Science 334: 1731-1734.

Kercmar J, Budefeld T, Grgurevic N, Tobet SA, Majdic G (2011). Adolescent social isolation changes social recognition in adult mice. Behav Brain Res 216: 647-651.

Kessler RC, Coccaro EF, Fava M, Jaeger S, Jin R, Walters E (2006). The prevalence and correlates of DSM-IV intermittent explosive disorder in the National Comorbidity Survey Replication. Arch Gen Psychiatry 63: 669-678.

Keyes KM, McLaughlin KA, Vo T, Galbraith T, Heimberg RG (2016). Anxious and aggressive: the co-occurrence of IED with anxiety disorders. Depress Anxiety 33: 101-111.

Lubin FD, Roth TL, Sweatt JD (2008). Epigenetic regulation of BDNF gene transcription in the consolidation of fear memory. J Neurosci 28: 10576-10586.

Marquez C, Poirier GL, Cordero MI, Larsen MH, Groner A, Marquis J et al (2013). Peripuberty stress leads to abnormal aggression, altered amygdala and orbitofrontal reactivity and increased prefrontal MAOA gene expression. Transl Psychiatry 3: e216.

Maya-Vetencourt JF, Tiraboschi E, Greco D, Restani L, Cerri C, Auvinen $\mathrm{P}$ et al (2012). Experience-dependent expression of NPAS4 regulates plasticity in adult visual cortex. J Physiol 590: 4777-4787.

Maya Vetencourt JF, Sale A, Viegi A, Baroncelli L, De Pasquale R, O'Leary OF et al (2008). The antidepressant fluoxetine restores plasticity in the adult visual cortex. Science 320: 385-388.

Maynard KR, Hill JL, Calcaterra NE, Palko ME, Kardian A, Paredes D et al (2016). Functional role of BDNF production from unique promoters in aggression and serotonin signaling. Neuropsychopharmacology 41: 1943-1955.

Miczek KA, de Boer SF, Haller J (2013). Excessive aggression as model of violence: a critical evaluation of current preclinical methods. Psychopharmacology 226: 445-458.

Nowicka D, Soulsby S, Skangiel-Kramska J, Glazewski S (2009). Parvalbumin-containing neurons, perineuronal nets and experiencedependent plasticity in murine barrel cortex. Eur J Neurosci 30: 2053-2063.

Onishchenko N, Karpova N, Sabri F, Castren E, Ceccatelli S (2008). Long-lasting depression-like behavior and epigenetic changes of BDNF gene expression induced by perinatal exposure to methylmercury. J Neurochem 106: 1378-1387.

Pascual R, Zamora-Leon SP, Valero-Cabre A (2006). Effects of postweaning social isolation and re-socialization on the expression of vasoactive intestinal peptide (VIP) and dendritic development in the medial prefrontal cortex of the rat. Acta Neurobiol Exp 66: 7-14.

Pruunsild P, Sepp M, Orav E, Koppel I, Timmusk T (2011). Identification of cis-elements and transcription factors regulating neuronal activity-dependent transcription of human BDNF gene. J Neurosci 31: 3295-3308.

Rosas-Vidal LE, Do-Monte FH, Sotres-Bayon F, Quirk GJ (2014). Hippocampal-prefrontal BDNF and memory for fear extinction. Neuropsychopharmacology 39: 2161-2169.

Saarelainen T, Lukkarinen JA, Koponen S, Grohn OH, Jolkkonen J, Koponen E et al (2000). Transgenic mice overexpressing truncated trkB neurotrophin receptors in neurons show increased susceptibility to cortical injury after focal cerebral ischemia. Mol Cell Neurosci 16: 87-96.

Sakata K, Woo NH, Martinowich K, Greene JS, Schloesser RJ, Shen L et al (2009). Critical role of promoter IV-driven BDNF transcription in GABAergic transmission and synaptic plasticity in the prefrontal cortex. Proc Natl Acad Sci USA 106: 5942-5947.

Sale A, Berardi N, Maffei L (2009). Enrich the environment to empower the brain. Trends Neurosci 32: 233-239.

Sales AJ, Joca SR (2016). Effects of DNA methylation inhibitors and conventional antidepressants on mice behaviour and brain DNA methylation levels. Acta Neuropsychiatr 28: 11-22.

Sandi C, Haller J (2015). Stress and the social brain: behavioural effects and neurobiological mechanisms. Nat Rev Neurosci 16: 290-304.

Senn V, Wolff SB, Herry C, Grenier F, Ehrlich I, Grundemann J et al (2014). Long-range connectivity defines behavioral specificity of amygdala neurons. Neuron 81: 428-437.

Shepard R, Heslin K, Coutellier L (2017). The transcription factor Npas4 contributes to adolescent development of prefrontal inhibitory circuits, and to cognitive and emotional functions: implications for neuropsychiatric disorders. Neurobiol Dis 99: 36-46.

Siegel A, Bhatt S, Bhatt R, Zalcman SS (2007). The neurobiological bases for development of pharmacological treatments of aggressive disorders. Curr Neuropharmacol 5: 135-147.

Siever LJ (2008). Neurobiology of aggression and violence. Am J Psychiatry 165: 429-442.

Society BP (2013). In: Antisocial Behaviour and Conduct Disorders in Children and Young People: Recognition, Intervention and Management. National Institute for Health and Care Excellence: Clinical Guidelines: Leicester, UK.

Sotres-Bayon F, Sierra-Mercado D, Pardilla-Delgado E, Quirk GJ (2012). Gating of fear in prelimbic cortex by hippocampal and amygdala inputs. Neuron 76: 804-812.

Spellman T, Rigotti M, Ahmari SE, Fusi S, Gogos JA, Gordon JA (2015). Hippocampal-prefrontal input supports spatial encoding in working memory. Nature 522: 309-314.

Tadic A, Muller-Engling L, Schlicht KF, Kotsiari A, Dreimuller N, Kleimann A et al (2014). Methylation of the promoter of brainderived neurotrophic factor exon IV and antidepressant response in major depression. Mol Psychiatry 19: 281-283.

Takahashi A, Nagayasu K, Nishitani N, Kaneko S, Koide T (2014). Control of intermale aggression by medial prefrontal cortex activation in the mouse. PLOS ONE 9: e94657.

Tiraboschi E, Guirado R, Greco D, Auvinen P, Maya-Vetencourt JF, Maffei L et al (2013). Gene expression patterns underlying the reinstatement of plasticity in the adult visual system. Neural Plasticity 2013: 605079.

Toffoli LV, Rodrigues GM Jr, Oliveira JF, Silva AS, Moreira EG, Pelosi GG et al (2014). Maternal exposure to fluoxetine during gestation and lactation affects the DNA methylation programming of rat's offspring: modulation by folic acid supplementation. Behav Brain Res 265: 142-147.

Toth M, Halasz J, Mikics E, Barsy B, Haller J (2008). Early social deprivation induces disturbed social communication and violent aggression in adulthood. Behav Neurosci 122: 849-854. 
Toth M, Mikics E, Tulogdi A, Aliczki M, Haller J (2011). Postweaning social isolation induces abnormal forms of aggression in conjunction with increased glucocorticoid and autonomic stress responses. Horm Behav 60: 28-36.

Tulogdi A, Toth M, Barsvari B, Biro L, Mikics E, Haller J (2014). Effects of resocialization on post-weaning social isolation-induced abnormal aggression and social deficits in rats. Dev Psychobiol 56: $49-57$.

Uchida S, Hara K, Kobayashi A, Otsuki K, Yamagata H, Hobara T et al (2011). Epigenetic status of Gdnf in the ventral striatum determines susceptibility and adaptation to daily stressful events. Neuron 69: 359-372.
Weiss B, Caron A, Ball S, Tapp J, Johnson M, Weisz JR (2005). Iatrogenic effects of group treatment for antisocial youths. J Consult Clin Psychol 73: 1036-1044.

Whitaker LR, Degoulet M, Morikawa H (2013). Social deprivation enhances VTA synaptic plasticity and drug-induced contextual learning. Neuron 77: 335-345.

Woolfenden S, Williams KJ, Peat J (2001). Family and parenting interventions in children and adolescents with conduct disorder and delinquency aged 10-17. Cochrane Database Syst Rev 2: CD003015.

Wright IK, Upton N, Marsden CA (1991). Resocialisation of isolation-reared rats does not alter their anxiogenic profile on the elevated X-maze model of anxiety. Physiol Behav 50: 1129-1132.

Supplementary Information accompanies the paper on the Neuropsychopharmacology website (http://www.nature.com/npp) 